\title{
ENABLING TECHNOLOGY IN THREE PRIMARY 5G SERVICES
}

\author{
Zoran Bojković, \\ Dragorad Milovanović, \\ Vladan Pantović
}

University of Belgrade, Belgrade, Serbia
Correspondence:

Zoran Bojković

e-mail:

z.bojkovic@yahoo.com

\begin{abstract}
:
This paper presents enabling technology in three primary $5 \mathrm{G}$ services, Internet of Things (IoT), ultra-reliable low- latency communication and mobile broadband multimedia. Starting from the fact that $5 \mathrm{G}$ massive connectivity has a huge influence - from infrastructure to business models, characteristics of IoT applications within significant parameters are reviewed together with Massive and Critical IoT services. Secondly, ultra-reliable low-latency services are invoked. The fundamental tradeoffs in terms of reliability-latency-throughput are outlined. Finally, mobile broadband, which is oriented to the service in human user access to multimedia content, concludes this presentation.
\end{abstract}

Keywords:

massive and critical IoT, ultra-reliable low-latency communication, enhanced mobile broadband.

\section{INTRODUCTION}

The coming fifth generation $(5 \mathrm{G})$ era has a huge influence on both the personal broadband mobile communications and the industry utilizations. According to the timetable defined in International Union (ITU), the proposals for $5 \mathrm{G}$ systems are to be submitted in 2019, and they will then be evaluated to make final IMT-2020 specifications by the end of 2020. The evolution is based on certain evaluation criteria including Key Performance Indicators (KPIs). The different usage scenarios of the $5 \mathrm{G}$ will set different profiles for the KPIs. For example, the key new factors brought by the $5 \mathrm{G}$ systems such as mm-Wave and 5G New Radio highly impact those KPIs. The exploratory phase is winding down as the $5 \mathrm{G}$ standardization phase already started in 2016. Standards are expected to be approved by ITU during the 2019-2020 timeframe and to become operational in the early 2020's. This way, 5G technology will become the key driving force behind the shift towards digitalization in all industries and a more intelligent, connected world. This has brought challenges to radio access and other networks. Technology and spectrum innovations have to meet divergent requirements within an integrated unified 5G standard framework [1]. 
The NGMN Alliance defines the following requirements that a $5 \mathrm{G}$ standard should fulfill such as: data rates of $100 \mathrm{Mbps}$ for metropolitan areas, 1 Gbps simultaneously to many users on the same office floor, several hundreds of thousands of simultaneous connections for sensors, significantly enhanced efficiency compared to $4 \mathrm{G}$, improved coverage, enhanced signaling efficiency, significantly reduced latency compared to Long Term Evaluation (LTE). LTE comes with OFDM-based air interface and all-IP network to provide many improvements. The demand for mobile data continues to grow, while LTEAdvanced and LTE-Advanced Pro promise higher technologies such as: carrier aggregation, coordinated multipoint, enhanced multi-antenna capabilities, relaying and improved inter-cell interference coordination for not only LTE cells, but also small cells and HetNets.

It is imperative that both the academia and industry participate in 5G technology roadmap activity for future networks and massive connectivity. As for participants, they are allowed to realize crucial benefits characterized by: optimized investment strategies for research and development, enabling visibility into future technology trends, focusing of efforts toward future solutions so that benefits are maximized for the industry, solutions that can be implemented in collaborative environments as well as in a competitive domain, exploring unique innovations to provide potential solutions, providing valuable input for the formulation of standards.

In order to study $5 \mathrm{G}$ enabling technology and primary services, this paper is structured as follows. After presenting technology enhancements, IoT as a key service together with the impact on $5 \mathrm{G}$ is outlined. Next, $5 \mathrm{G}$ ultra reliable and low-latency communication services are emphasized. The final part is devoted to the implementation of 5G mobile broadband technology in multimedia services.

\section{TECHNOLOGY ENHANCEMENTS}

In order to avoid misconceptions about 5G technology, a collaboration between experts in academia, the industry as well as standard development organizations becomes an obligation. The goal is to identify challenges for technologies, paving the way for providing wireless communications with massive connectivity, tactile Internet, quality of service and network slicing. On the other hand, 5G is called to produce a complete revolution in the way users can access content and services in their general daily activities, such as: ultra-high system capacity, massive device connectivity, ultra-low latency, low-cost devices and diverse service types. To satisfy these requirements, enhanced technologies are necessary: massive MIMO, mm-Wave, ultra-dense networks, non-orthogonal multiple-access (NOMA).

Massive MIMO requires the simultaneous processing of signals from many antenna chains and computational operations. MIMO is considered to be one of the key technologies in $5 \mathrm{G}$ systems due to its advantages of improving both the received signals-to-noise ratio (SNR) and the bandwidth efficiency. Massive MIMO is capable of substantially increasing both the capacity as well as the energy efficiency [2]. Recent advances on system-algorithm-hardware co-design have led to extremely energy-efficient implementations.

WiFi systems are the most widely deployed wireless technologies. There are actually very sophisticated systems that were created to satisfy difficult and challenging requirements. For example, they operate in unlicensed bands with very severe emission limitations. Also, they need to provide high data rates because they compete with wired LAN-using technologies that link the Ethernet with super-fast rates. Furthermore, in some scenarios, WiFi systems are deployed in a somewhat dispersed manner, without strong centralized control. Thus, challenges of interference and resource sharing arise. Different types of traffic might need different adjustments to meet the Quality of Service (QoS), which could generate additional challenges from the resource sharing and interference management perspectives. These other technical challenges have been handled over the years through a variety of solutions and approaches. Examples of such creative solutions include the various coordination functions at the MAC layer spread spectrum, OFDM, etc. to handle challenging constraints at the physical layer while trying to achieve high data rates.

Dense HetNets are one of the $5 \mathrm{G}$ technologies capable of significantly improving the network capacity. The core idea of HetNets is essentially to move the low power Base Stations (BSs) closer to the users in order to form small cells [3]. The basic idea of massive MIMO-assisted HetNets is to install hundreds or thousands of antennas at the macro BSs for offering an unprecedented level of spatial degree of freedom, while using a single antenna at the densely positioned small cell BSs.

The fundamental concept of NOMA (Non-Orthogonal Multiple Access) facilitates supporting multiple users in the power domain. NOMA uses a new dimension to perform multiplexing within one of the timefrequency domains. In fact, NOMA can be regarded as a technique that has the promising potential of facilitating 
integration with the existing legacy solutions [4]. One of the main challenges of NOMA is the compatibility with other technologies towards $5 \mathrm{G}$ and beyond. Several key directions have been identified: ultra-densification, massive MIMO arrangements, full duplex solutions, cloud-based Radio Access Networks (RANs), mm Wave communications, Device-to-Device (D2D) and Machine-to-Machine (M2M) communications, energy harvesting, virtualization and softwarization.

The 5G will support a wide range of features and use cases, going well beyond current cellular networks. Increased capacity to support Enhanced Mobile Broadband (eMBB) services is a key driver for $5 \mathrm{G}$. Service
eMBB is targeting high data rates, such as seamless data access both indoors and outdoors, high-resolution multimedia streaming in high mobility scenarios. $5 \mathrm{G}$ is also required to support Ultra Reliable Low-Latency Communications (uRLLC) and Massive Machine Type Communications (mMTC). uRLLC are defined as applications that have stringent latency and reliability requirements (vehicular communications and control network industry), while mMTC is the basis for connectivity in the Internet of Things (IoT) including infrastructure management, environment monitoring, and healthcare. The basic characteristics of mMTC, uRLLC and eMBB service categories are presented in Table 1.

Table 1. Basic characteristics of mMTC, uRLLC and eMBB primary service categories

\begin{tabular}{|c|c|c|c|}
\hline Category & mMTC & uRLLC & eMBB \\
\hline $\begin{array}{l}\text { Main } \\
\text { motivation }\end{array}$ & $\begin{array}{c}\text { Increase the number of } \\
\text { support devices and support } \\
\text { low-cost devices }\end{array}$ & $\begin{array}{l}\text { Reduce end-to-end latency } \\
\text { and increase robustness of } \\
\text { data transmission }\end{array}$ & $\begin{array}{l}\text { Increase spectral efficiency } \\
\text { and increase peak throughput }\end{array}$ \\
\hline $\begin{array}{l}\text { Key } \\
\text { requirements }\end{array}$ & $\begin{array}{l}1 \times 10^{6} \text { devices } / \mathrm{km}^{2} \\
100 \mathrm{x} \text { energy efficiency }\end{array}$ & $\begin{array}{l}\text { 10ms end-to-end latency } \\
(0.5 \mathrm{~ms} \text { physical-layer }) \\
\text { BLER }<10^{-5} \text { within } 1 \mathrm{~ms}\end{array}$ & $\begin{array}{l}\text { 20x peak throughput, 100x } \\
\text { area throughput, } 5 x \text { spectral } \\
\text { efficiency }\end{array}$ \\
\hline Packet size & $100 x$ bytes & $10-100$ of bytes & $>>100$ bytes \\
\hline $\begin{array}{l}\text { Physical-layer } \\
\text { solutions }\end{array}$ & $\begin{array}{l}\text { Non-orthogonal multiple } \\
\text { access, overloaded data } \\
\text { transmission, active user- } \\
\text { detection }\end{array}$ & $\begin{array}{c}\text { Instant access, errorless data } \\
\text { transmission }\end{array}$ & $\begin{array}{c}\text { Massive antennas, mmWave } \\
\text { band-aggregation, and new } \\
\text { waveforms }\end{array}$ \\
\hline Applications & $\begin{array}{l}\text { Logging, metering, lodging, } \\
\text { tagging, and measuring }\end{array}$ & $\begin{array}{l}\text { Tactile Internet, remote } \\
\text { control, self-car driving, } \\
\text { augment reality, industrial } \\
\text { automation }\end{array}$ & $\begin{array}{l}\text { Virtual reality, real-time } \\
\text { security, 3D image, and } 4 \mathrm{~K} \\
\text { video streaming }\end{array}$ \\
\hline
\end{tabular}

\section{PRIMARY 5G SERVICE CATEGORIES}

The use case scenarios for the $5 \mathrm{G}$ mobile wireless networks involve IoT, ultra-reliable and low-delay communication as well as multimedia broadband services.

\section{IoT massive connectivity}

One of the primary goals of $5 \mathrm{G}$ wireless networking is the integration of heterogeneous access technologies that facilitate the connectivity of physical or virtual things with the Internet. IoT enables data communication between heterogeneous devices with modest or no personal intervention. The goal of IoT is to move towards more autonomous, scalable, connected and location-independent infrastructure. IoT presents service management with more challenging issues. IoT applications can be divided into two major categories: Massive IoT and Critical IoT applications. Massive applications have specific requirements of extended coverage area and high scalability, low-energy consumption and low-cost user equipment [5]. Examples of Critical IoT applications include healthcare systems, traffic and industrial control, Smart Grid. Also, lower latency for acceptable end-user experience as well as higher availability and reliability are desirable [6]. IoT applications have varying requirements in diverse domains: smart home, smart city, intelligent transportation system, data reliability in the industrial IoT. An outline of the differences in requirements is presented in Table 2. The characteristics are the application domain, tolerable delay, reporting intervals, and data rate. 
Design engineers can easily find which one of the offered alternatives is best suited for current IoT use case. Key design requirements support massive devices, extended coverage, network cost, long battery life, compactness and data reliability, security and privacy. For example, the total cost of devices should be as low as possible to support massive deployment. It implies the reduction in the device complexity and production cost. Also, the total cost of network connectivity should be kept at a minimum in massive IoT deployment. Most IoT devices operate for a long-term period without any human intervention. Thus, the energy efficient design of hardware and software is required. In the case of massive IoT connectivity, extended coverage design is also important. The location and the end user's identity should not be accessible to the public.

Table 2. Short overview of IoT technical parameters

\begin{tabular}{|c|c|c|c|}
\hline Application & $\begin{array}{c}\text { Tolerable } \\
\text { delay }\end{array}$ & $\begin{array}{l}\text { Update } \\
\text { frequency }\end{array}$ & $\begin{array}{l}\text { Data } \\
\text { rate }\end{array}$ \\
\hline Structural health & $30 \mathrm{~min}$ & $10 \mathrm{~min}$ & low \\
\hline $\begin{array}{l}\text { Waste } \\
\text { management }\end{array}$ & $30 \mathrm{~min}$ & 1 hour & low \\
\hline Video surveillance & seconds & real-time & high \\
\hline $\begin{array}{l}\text { Air quality } \\
\text { monitoring }\end{array}$ & $5 \mathrm{~min}$ & $30 \mathrm{~min}$ & low \\
\hline $\begin{array}{l}\text { Monitoring and } \\
\text { supervision }\end{array}$ & $\begin{array}{l}\text { seconds or } \\
\text { millisec- } \\
\text { onds }\end{array}$ & seconds & low \\
\hline $\begin{array}{l}\text { Closed loop } \\
\text { control }\end{array}$ & $\begin{array}{l}\text { millisec- } \\
\text { onds }\end{array}$ & $\begin{array}{l}\text { millisec- } \\
\text { onds }\end{array}$ & low \\
\hline $\begin{array}{l}\text { Interlocking and } \\
\text { control }\end{array}$ & $\begin{array}{l}\text { millisec- } \\
\text { onds }\end{array}$ & $\begin{array}{l}\text { millisec- } \\
\text { onds }\end{array}$ & low \\
\hline $\begin{array}{l}\text { Patient's health } \\
\text { delivery and } \\
\text { monitoring }\end{array}$ & $\begin{array}{c}\text { low } \\
\text { (seconds) }\end{array}$ & $\begin{array}{l}1 \text { report per } \\
\text { hour/day }\end{array}$ & high \\
\hline $\begin{array}{l}\text { Real-time emer- } \\
\text { gency response } \\
\text { and remote diag- } \\
\text { nostics }\end{array}$ & $\begin{array}{c}\text { low } \\
\text { (seconds) }\end{array}$ & $\begin{array}{l}\text { requires } \\
\text { ad-hoc } \\
\text { emergency } \\
\text { communi- } \\
\text { cation }\end{array}$ & high \\
\hline $\begin{array}{l}\text { Real-time man- } \\
\text { agement of infor- } \\
\text { mation in supply } \\
\text { chain }\end{array}$ & $\begin{array}{c}\text { low } \\
\text { (seconds) }\end{array}$ & $\begin{array}{l}1 \text { report per } \\
\text { hour/day }\end{array}$ & high \\
\hline
\end{tabular}

While massive IoT systems require improved system coverage and reduced terminal cost, critical IoT has small data packets and delay-intolerant communication.
As the $5 \mathrm{G}$ system is characterized by a high degree of heterogeneity using services, device classes, deployment types, environments and mobility levels, key enablers for these activities are based on: software-defined networking, network function virtualization, cognitive radio, scalability, network management and mobility.

Technology Software Defined Networking (SDN) separates the data and control planes, centralizes network control and allows programmability of the network by external applications [7]. In that way, reconfiguration is provided as a dynamic, flexible and automatic method. The most important issues involve avoiding a collapse of the network and guaranteeing that QoS. Technology Network Function Virtualization (NFV) is highly complementary to SDH. The main idea behind NFV is to virtualize a set of network functions implemented into software packages which are now available for the configuration of some services provided by the network. Both solutions (SDN and NFV) can be combined to achieve optimal performance for management of critical and massive IoT applications [8].

Technology Cognitive Radio (CR) utilizes the limited licensed spectrum resources in order to support new service requirements in $5 \mathrm{G}$ mobile networks for IoT applications. The CR operates in the best available channel using dynamic spectrum access techniques. This kind of technology provides the detection of users present in the spectrum sensing. Next, it identifies the available free spectrum, based on spectrum management, spectrum sharing and spectrum mobility.

By introducing new heterogeneous devices, applications and functions are based on scalability. For scale efficiency, optimization of the massive deployment of IoT has to be provided [9]. This way, network capacity becomes scalable to accommodate as many connected devices as possible. Efficient network management solutions provide monitoring, remote control and maintenance concerning network equipment, services and devices. The management functions in the IoT carry out remote tasks across a heterogeneous interconnected network. Such a system enables diagnostics in IoT devices and real-time control. This way, the cost of operation will be managed in terms of resource constrained networks. Network coverage remains an open research issue for effective deployment scenarios, since most of the massive and critical IoT users are mobile. As a result of user mobility, connected devices undergo service interruptions. This is the reason why an efficient mobility management mechanism is needed to control the connected devices in IoT networks. 
The use case scenario for the $5 \mathrm{G}$ mobile wireless networks involves eMBB human users accessing multimedia content, Massive Machine Type Communications (mMTC), a large number of connected devices, as well as uRLLC with the most stringent requirements. In this case, reliability is the probability that latency will not overstep the pre-determined deadline in data transmission. As for latency, it is defined as the delay of data packet measured in the same layer at the transmitter and at the receiver [10]. Simultaneous requirements for reliability, latency and throughput in a wireless system are demanding. The improvement in throughput and reliability cause the degradation in terms of latency. At the same time, the latency is increased due to an error control mechanism which implies some overhead. The diagram of reliability vs. latency in wireless communication is shown in Figure 1.

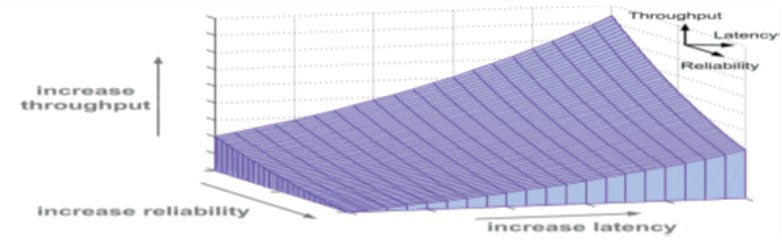

Fig. 1. The diagram of reliability vs.

latency in a wireless communication

The effective bandwidth function is the minimum service rate assuming prescribed latency requirements in a tradeoff of throughput vs. latency. The effective capacity is the maximum constant data rate that a given service supports assuming the delay constraints [11].

The total latency in the $5 \mathrm{G}$ system is contributed by user plane and control plane. The user plane latency is measured in transmission and reception of a packet at the corresponding network layers. The control plane latency represents the transition time from an idle state to an active state when user equipment establishes a radio resource control connection.

It is necessary to transmit an uRLLC data packet as soon as it is received at the node / base station on the Downlink (DL) or generated by the User Equipment (UE) on the Uplink (UL). Autonomous transmission is also one of the solutions, where UE transmits on pre-defined uplink resources without the need for an explicit grant from the node. The uRLLC challenges cover not only the physical layer but also all aspects of the $5 \mathrm{G}$ system design.

\section{Mobile broadband technology}

5G wireless networks are a combination of different, advanced technologies designed to support Gbps data rates, massive capacity and connectivity as well as widely different network deployment scenarios. Significantly lower end-to-end latencies and higher connectivity reliability are needed in real-time applications with Quality of Experience (QoE) guarantees. Also, the reduction of energy consumption by orders of magnitude is required [12].

Mobile broadband is a significant multimedia delivery platform. Thus, it is important to study technical operational and regulatory issues. Considering current and future requirements and constraints, it is important to research feasible and beneficial use cases and usage platforms. A point-to-multipoint interface Multimedia Broadcast Multicast Service (MBMS) is specified to provide efficient delivery of broadcasting services in the core and the radio access networks. The service defines transmission via Single-Frequency Network (SFN) configurations for a broadband transmission across multiple calls. Examples of applications are mobile TV and radio broadcasting, live streaming video services, as well as file delivery and emerging alerts.

The work on adding broadcast/multicast support to $3 \mathrm{G}$ networks began in 2002 when $3 \mathrm{GPP}$ created a work item in GSM/UMTS and CDMA2000. Requirements are defined for the optimization of the distribution of video traffic (3GPP TS25.992 MBMS UTRAN/GERAN Requirements, Oct. 2003). To support Ultra-HDTV, Realize 14 upgraded media codecs and formats to values specified for broadcast television. HDR (High Dynamic Range) format support was added in Release 15 for improvement in MBMS services.

Higher picture quality is a general trend for all services and devices. Service providers seek to deliver as high a quality as possible, despite the growing associated costs and the issues related to network capacity. TV picture quality technical parameters are spatial resolution (the number of pixels in a picture), temporal resolution (the number of picture frames per second), color gamut (the range of colors that can be displayed) and dynamic range (the span between minimum and maximum brightness). The combination of parameters is standardized in order to ensure interoperability. The picture formats that indicate progressively higher quality are SDTV, HDTV and UHDTV. Examples of multimedia services include on-demand, time shifted, interactive and personalized services, multi-screen and multi-view 
services and cross-platform services. In order to enable eMBB in mission critical low-latency video applications via cost-efficient RANs for $5 \mathrm{G}$ Phase 2 , the focus is on mm-Wave access/backhauling with MEC (Mobile Edge Computing) in uHSLLC service [13].

\section{CONCLUSION}

Looking back at the evolution of technology, there is a separation of 10 years from one generation of mobile system to the next, both if one takes as a reference point the first trials, the first deployment, the mass market uptake or even its obsoletes. Based on this, we will have real mass market adoption of 5G around 2025. However, for the future $6 \mathrm{G}$ technology development, the forecast could be based on the law of accelerated returns stating that evolution is accelerating and what used to take 10 years will take 7 years, and then just 5 years, and so on. Collaboration between experts in academia, the industry and standard development organizations has become an obligation.

The goal of this paper is to study primary service categories in typical 5G use cases. Special interest is given to IoT connectivity technology, starting from the point that massive machine type communication requires a significant network capacity increase. As mobile wireless systems also address uRLLC, it is a challenging technological task to reduce the latency, while simultaneously providing certain levels of reliability. In order to enable eMBB services in mission critical low-latency video applications, a new class Ultra-High-Speed Low-Latency Communication (uHSLLC) service is introduced. The corresponding architecture should include softwaredefined networking and network function virtualization to accommodate diverse use cases in an acceptable cost-efficient manner.

\section{REFERENCES}

[1] J.G. Andrews et al., "What will 5G be?," IEEE Journal SAC, vol. 32, pp. 1065-1082, June 2014.

[2] J. Li, X. Wu, R.Laroia, OFDMA Mobile Broadband Communications: A Systems Approach, Cambridge Uni. Press, 2013.

[3] A. Adhikary, H.S. Dhillon, G. Caire, "Massive MIMO mits HetNet: Interference coordination through spatial blanking," IEEE Journal SAC, vol. 33, pp. 1171-1186, June 2015.

[4] Y. Lin et al., "Non-orthogonal multiple access for 5G and beyond," Proc. of the IEEE, vol. 105, pp. 2347-2381, Dec. 2017.

[5] J. Gubbi et al., "Internet of Things (IoT): A vision, architectural elements and future directions", Journal Future generation computer systems, vol. 29, pp.1645-1660, Sept. 2013.

[6] A. Ali et al., "Technologies and challenges in developing machine-to-machine applications: A survey", Journal of Network and computer applications, vol. 83, pp. 124-139, Feb. 2017.

[7] D. Kreutz et al., "Software-defined networking: A comprehensive survey", Proc. of the IEEE, vol.103, pp. 14-76, 2015.

[8] I.F. Akyldiz, S.C. Lin, P. Wang, "Wireless softwaredefined networks (W-SDN) and network function virtualization (NFV) for $5 \mathrm{G}$ cellular systems: An overview and qualitative evaluation", Computer networks, vol.93, pp.66-79, 2015.

[9] I. Ishaq et al., "IETF standardization in field of Internet of Things (IoT): A survey", Journal Sensor\&actuators networks, vol.2, pp.235-287, 2013.

[10] G. Pocovi et al., "Achieving ultra-reliable low-latency communications: Challenges and envisioned system enhancements", IEEE Network, vol.32, pp.8-15, 2018.

[11] B. Soret et al., "Fundamental tradeoffs among reliability, latency and throughput in cellular networks", in Proc. IEEE Globecom 2014, pp.1391-1396

[12] E. Liotou et al., "Quality of Experience management in mobile cellular networks: Key issues and design challenges", IEEE Comm. Magazine, vol.53, pp.145153, July 2015.

[13] MG-MiEdge Project (available online https://5gmiedge.eu/) 